\title{
Simulación de un Proceso de Fabricación de Bicicletas. Aplicación Didáctica en la Enseñanza de la Ingeniería Industrial
}

\author{
Yesid Forero-Páez ${ }^{(1,2)}$ y Jaime A. Giraldo(1) \\ (1) Facultad de Ingeniería, Departamento de Ingeniería Industrial, Univ. Nacional de Colombia, \\ Carrera 27 \# 64-60, Manizales-Colombia (e-mail: yforero@autonoma.edu.co, jaiagiraldog@unal.edu.co) \\ (2) Facultad de Ingeniería, Departamento de Mecánica y Producción, Universidad Autónoma de \\ Manizales, Antigua estación del ferrocarril, Manizales-Colombia
}

Recibido Sep. 24, 2015; Aceptado Nov. 19, 2015; Versión final Dic. 19, 2015, Publicado Jun. 2016

\begin{abstract}
Resumen
Se presentan los resultados obtenidos con el uso de un modelo de simulación de un proceso de fabricación de bicicletas en un curso ingeniería industrial. La interacción con el modelo permite a los estudiantes comprender las principales relaciones causa-efecto de dichos procesos. Dicha interacción se da a través de hojas electrónicas, mediante las cuales el estudiante establece los valores (determinísticos o aleatorios) de un conjunto de variables de decisión (causas): tiempos de operación, programa de adquisición de materia prima, y parámetros de mantenimiento preventivo/correctivo que el estudiante considera apropiado para satisfacer un nivel determinado de demanda de bicicletas y el uso de la capacidad de producción (variables efecto). Se muestran los resultados de simulación de dos situaciones de operación del sistema y se compara el desempeño de este en términos del número de bicicletas fabricadas y las tasas de utilización de tres áreas del proceso. Se concluye que el uso del modelo representa un soporte didáctico apropiado en asignaturas relacionadas con la gestión de sistemas de producción industrial.
\end{abstract}

Palabras clave: simulación; sistema de producción; tiempos de operación; capacidad de producción; ingeniería industrial; didáctica

\section{Simulation of a Bicycle Manufacturing Process. Didactic Application in the Teaching of Industrial Engineering}

\begin{abstract}
The results obtained with the use of a simulation model of a process of bicycle manufacturing in a course of industrial engineering. The interaction with the model allowed students to understand the main causeeffect relations in such processes. Such interaction, is done through the use of spreadsheets, where students establish the values (deterministic or random) of a set of decision variables (causes): operating times, program acquisition of raw materials, and preventive/corrective maintenance parameters that the student considers appropriate to satisfy a certain level of demand for bicycles and the use of the production capacity (variable effect). The results of two situations obtained with the model are compared in terms of the number of bicycles manufactured and the utilization rates of the three areas of the process. It is concluded that the use of the model represents an appropriate didactic tools for the teaching of subjects related to management of industrial production systems.
\end{abstract}

Keywords: simulation; production system; operating times; production capacity; industrial engineering; didactics 


\section{INTRODUCCIÓN}

La evolución de la sociedad en la segunda mitad del siglo XX y lo corrido del nuevo milenio, ha generado profundas transformaciones en diversos aspectos. El sector educativo no ha sido ajeno a estos cambios y ha sido uno de los sectores más sensibles a las transformaciones derivadas de esta dinámica, requiriendo de acciones permanentes que lo pongan en sintonía con las demandas de la sociedad y el entorno de manera general; López y Valenti (2000) citado por Molina (2012) plantea que la sociedad postindustrial "avanza inexorablemente hacia la primacía de la inteligencia y el saber" como principales factores del progreso social y económico; la educación no puede ser ajena a este entorno y debe procurar estrategias permanentes de cambio y de adaptación a las nuevas condiciones.

Para Feo (2010) una estrategia didáctica es el conjunto de métodos, técnicas y actividades a través de los cuales el docente y los discentes, desarrollan el proceso enseñanza-aprendizaje, adaptándose a las necesidades de los participantes de manera significativa. Ferreiro (2004) considera que una estrategia de enseñanza es un conjunto de procedimientos empleados por el docente para hacer posible el aprendizaje del discente, los cuales incluyen operaciones físicas y mentales para facilitar la confrontación del sujeto que aprende con el objetivo del conocimiento. Paralelamente define una estrategia de aprendizaje como "... los procedimientos mentales que el estudiante sigue para aprender. Es una secuencia de operaciones cognoscitivas y procedimentales que el estudiante desarrolla para procesar la información y aprender significativamente".

Laborí (2011) evidencia el hecho de que en ingeniería, el método tradicional de enseñanza se ha basado básicamente, en la clase magistral impartida por el profesor, donde el estudiante puede hacer las preguntas deseadas y tomar apuntes de lo dicho, luego, con esos apuntes, libros de texto y consulta convencionales, en una palabra con información "impresa", - procedía al estudio individual, apoyado en la realización de talleres conceptuales que reafirmen los puntos tratados desde la teoría, buscando aplicaciones prácticas; sin embargo como lo propone (Troye Nordkvellei, 2004), la relación didáctica- tecnología “...con el advenimiento de la tecnología electrónica, la influencia de la tecnología en la educación se ha convertido en inmensa.", de la misma manera Torres (2010) citando Pozo y Monereo (1999) manifiesta el hecho de que en los últimos años, las estrategias de aprendizaje han ido cobrando una importancia cada vez mayor, tanto en la investigación psicológica como en la práctica educativa, que ha venido a convertir el aprender a aprender en una de las metas fundamentales de cualquier proyecto educativo. Como lo menciona Fernández \& Duarte (2013) citando a (Vacca et al., 2011; Alejandro, 2004; Ertugrul, 2000; Okutsu et al., 2013), las diversas metodologías de enseñanza "...se han visto favorecidas con el uso de las Tecnologías de la Información y la Comunicación, TIC, las cuales, en el caso de la ingeniería, implican la utilización de plataformas en internet para el desarrollo de actividades de formación, laboratorios virtuales y experimentación remota, interfaces web para visualizar contenidos, junto con herramientas de simulación específicamente diseñadas para desarrollar habilidades y destrezas en los futuros ingenieros".

Se considera de otra parte, el hecho que la evolución de los procesos de enseñanza -aprendizaje, de la mano del desarrollo tecnológico en información y comunicaciones (TIC), requiere que los procesos educativos trasciendan de la relación docente - estudiante basada en la transmisión de conocimientos, hacia una enseñanza participativa de los estudiantes, tal como se plantea en (AUNIES, 2001). De otro lado (Holvikiki, 2007) considera que "la educación superior deberá implementar el paradigma de la educación permanente que implica dotar a los estudiantes de una disciplina intelectual bien cimentada para el autoaprendizaje en las diversas situaciones en que se encuentre". Considerando que dicho autoaprendizaje puede ser soportado en TIC, la UNESCO (2009), dentro de los principios propugnados en materia del uso de las TIC en educación establece en algunos de sus apartes que: i) Se debe mantener un equilibrio en el uso de las nuevas tecnologías y las tecnologías convencionales, ii) La demanda por educación superior no podrá ser satisfecha en el mundo desarrollado ni en el mundo en vías de desarrollo sin la contribución de la modalidad de aprendizaje virtual o a distancia y, iii) Las necesidades de formación profesional no podrán cumplirse sin el apoyo de clases virtuales, laboratorios virtuales, etc.

En este último sentido, Fredes et al (2012) citando a (Helsel, 1992) describen los ambientes virtuales como un espacio que permite "... a los usuarios convertirse en participantes de un mundo abstracto, donde la posición física del objeto no existe", señalando además que una categoría de software que recrea ambientes virtuales para uso en el aprendizaje la constituye los simuladores. De hecho, la simulación por computadora ha sido una de las áreas de la investigación de operaciones, que debido a su potencial de uso y al avance en tecnología informática, ha ido penetrando lentamente en el quehacer del ingeniero industrial, constituyéndose en una herramienta supremamente útil para el análisis de sistemas de producción. Al respecto, (Chung, 2003) anota que: "El objetivo del modelo de simulación consiste precisamente, en comprender, analizar y mejorar las condiciones de operación relevantes al sistema, su actualidad y la evolución de la tecnología". 
La mayoría de sistemas de producción y sus procesos asociados exhiben alta complejidad en su operación y diseño. Para Harrell (2004), esta complejidad se da en razón a tres factores: a) Alta interdependencia entre los componentes del sistema, b) Comportamiento aleatorio y c) Necesidad de estudiar el desempeño del sistema en el tiempo. Dados estos factores, de seguro no es fácil para un docente en ingeniería industrial lograr que sus discentes comprendan la dinámica de estos sistemas/procesos, conllevándolo a responder algunos interrogantes desde el punto de vista didáctico: ¿Cómo representar la interdependencia entre los diferentes procesos de fabricación? ¿Cómo modelar matemáticamente la variación aleatoria que se da en los procesos? ¿Cómo modelar los muy diversos eventos que se dan en el tiempo en un ambiente de fabricación? ¿Cómo medir el desempeño del sistema a partir de un modelo simulado del sistema? ¿Cuál sería una metodología educativa apropiada?

Existen diversas metodologías que buscan acercar al estudiante a la solución de problemas del mundo real. Una de ellas es el Aprendizaje Basado en Problemas, (ABP), la cual permite centrar el aprendizaje en el estudiante, e introducir en la enseñanza problemas abiertos y más próximos a su desempeño profesional (ITESM, 2000; Restrepo, 2005) citado por Fernández \& Duarte (2013). Para Moran (2011), el Aprendizaje Basado en Problemas (ABP) es el método de mayor efectividad para aprender la profesión de la ingeniería, pues considera que el ingeniero es en esencia un solucionador de problemas; para actuar con eficacia, conceptualiza, diseña, implementa y opera servicios, productos y procesos que satisfacen necesidades sociales. El enfoque problémico, entendido como un sistema complejo integrado por propósitos educativos para plantear, buscar y dar solución a problemas disciplinares e interdisciplinares (Gómez, V. y Díaz, M. 2003) es abordado por Álvarez (1999) citado por Ortiz (2009), señalando que la esencia de la enseñanza problémica, consiste en que los estudiantes, bajo la tutoría del profesor se introducen en la búsqueda y solución de problemas nuevos para ellos, aprendiendo a emplear los conocimientos para resolverlos, a la vez que desarrolla habilidades para analizarlos, buscar información, proponer soluciones.

La resolución de problemas forma además a los estudiantes para adelantar procesos meta cognitivos necesarios para perfeccionar cada vez más las interacciones con el medio, potenciar la argumentación, el respeto por las posiciones de los otros, y el componente afectivo-emotivo por el conocimiento, por su uso y aplicación en la construcción de una sociedad mejor. De hecho en la gestión de procesos de producción industrial, se pueden plantear diversos problemas, en términos sencillos, sobre diversos aspectos de esta gestión (producto, plazo, costo, etc.) que requieren explicar aspectos como: cuanto y cuando producir, cantidad de mano de obra emplear, cantidad de materia prima requerida, parámetros de mantenimiento, etc.

Para Vizcarro y Juárez (2007), la idea básica según ABP, consiste en plantearle al discente un problema, que reúna los siguientes requisitos: 1) Que guarde relación con el conocimiento previo de los discentes y, al mismo tiempo, comprenda una serie de elementos desconocidos que demanden más información, 2) Que el problema sea interesante y relevante para los estudiantes, de manera que capte su atención, 3) Que haga que los estudiantes formulen objetivos de aprendizaje sobre la(s) materia(s) deseada(s) y 4) que el problema refleje la complejidad de los problemas de la vida real. Se espera que el estudiante evalué las posibles soluciones al problema planteado, mediante el modelo de simulación, el cual a través de una interface gráfica, fije los niveles de diversas variables de decisión que conlleven a un mejor desempeño del sistema representado. En consecuencia, en la búsqueda de información para dar solución al problema, el estudiante se verá obligado a indagar sobre los siguientes temas relacionados con los procesos de producción de bicicletas, entre otros: demanda independiente/dependiente; árbol de partes componentes de un producto; árbol de procesos para fabricar un producto; inventario de materia prima, producto en proceso y producto terminado; tiempos de proceso/suministro determinísticos y estocásticos; compras de materia prima; programación de producción; costos de producción, mantenimiento preventivo/correctivo, etc.

\section{MODELO DE SIMULACION Y DECISION}

Para la construcción/simulación del modelo se siguió la metodología propuesta por Giraldo (2014), con base en lo expuesto por Law y Kelton (2007), Banks et al. (2009), Shanon (1988), Gordon (1978). Las principales etapas se describen a continuación:

\section{Definición del sistema/proceso a modelar}

Se selecciona un proceso de fabricación de fácil comprensión, que le permita a quienes empleen el modelo un inmediato entendimiento de las fases de operación y un adecuado conocimiento de las partes involucradas en los procesos de ensamble; por esta razón se elige el proceso de elaboración de bicicletas, por ser un producto de uso cotidiano y de gran familiaridad en la población, especialmente entre los jóvenes. Inicialmente se hace recolección de información sobre el producto/proceso (ver Tabla 1 y Figura 1). 
Tabla 1: Estructura del producto y operaciones para su fabricación.

\begin{tabular}{|c|l|c|l|}
\hline \multicolumn{4}{|c|}{ Componentes } \\
\hline A & Tubo superior & Q & Sistema de tracción \\
\hline B & Tubo dirección & R & Rueda trasera \\
\hline C & Tubo Asiento & S & Manzana \\
\hline D & Tubo inferior & T & Piñones \\
\hline E & Vaina & U & Llanta \\
\hline F & Tija & V & Freno \\
\hline G & $\begin{array}{l}\text { Sistema de } \\
\text { transmisión }\end{array}$ & W & Rayo \\
\hline H & Cadena & X & Buje \\
\hline I & Estrella & Y & Rueda Delantera \\
\hline J & Pedal & Z & Soldadura \\
\hline K & Biela & A1 & Puntera \\
\hline L & Cambios & B1 & Amortiguador \\
\hline M & Sistema de dirección & C1 & Sillín \\
\hline N & Telescopio & D1 & Control Cambios \\
\hline O & Manubrio & E1 & Control Freno \\
\hline P & Tijera & & \\
\hline \multicolumn{5}{|l|}{} \\
\hline 1 & Corte & 5 & Pintura \\
\hline 2 & Doblado & 6 & Ensamble \\
\hline 3 & Pulido & 7 & Inspección \\
\hline 4 & Soldadura & Almacenamiento \\
\hline
\end{tabular}

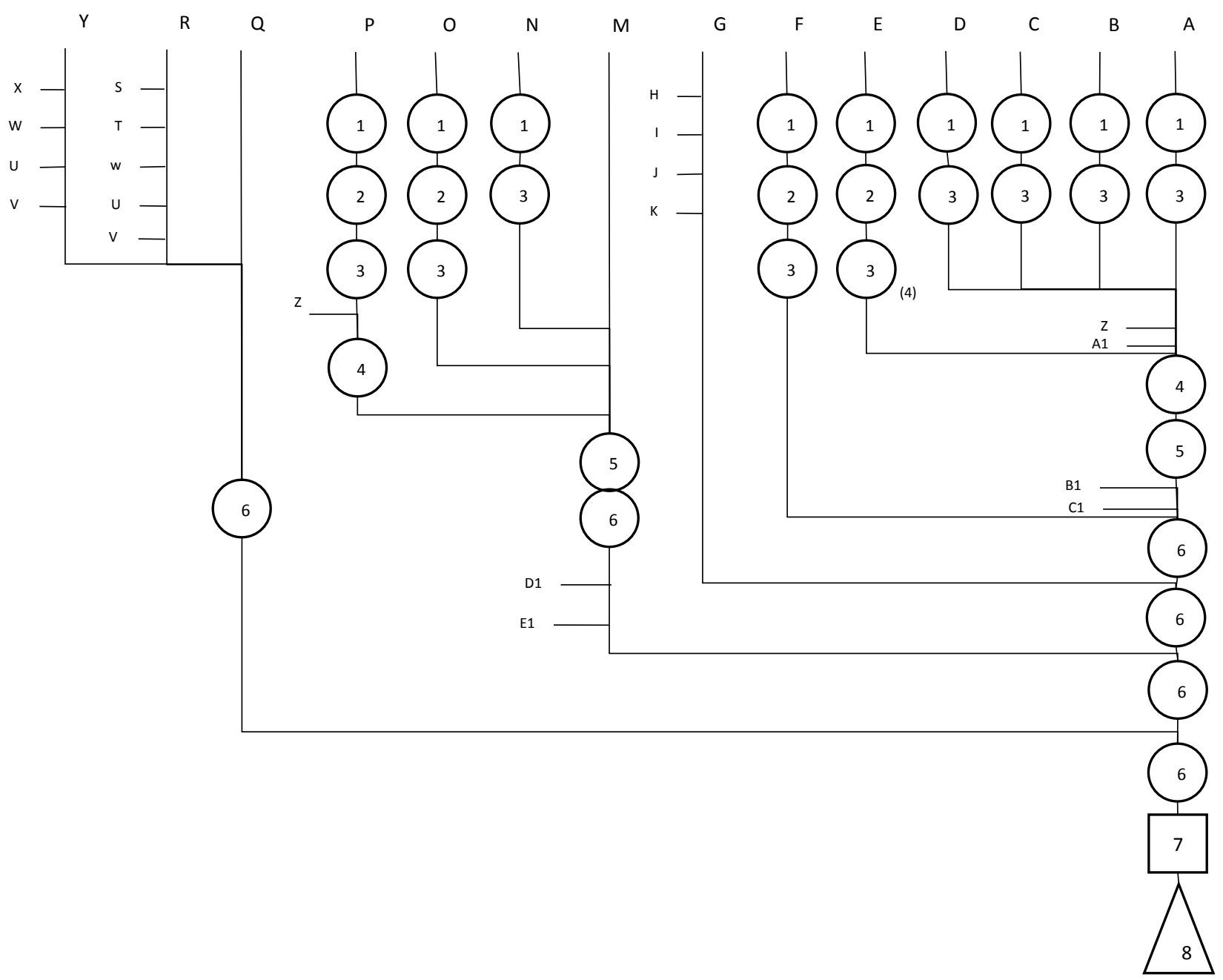

Fig. 1: Diagrama de precedencia del proceso de fabricación 
La estructura está determinada por un cuerpo central (marco) y unos componentes establecidos por sub ensambles que se van integrando al producto de acuerdo a la etapa de producción respectiva (Figura 1). El marco inicia su fabricación con el arribo de los tubos al área de almacenamiento, desde la cual es dirigido hacia el proceso de producción de marcos y manubrios, pasando por las operaciones de corte, doblado, soldadura y pintura; paralelamente a la fabricación del marco, se van realizando sub ensambles que de acuerdo a la fase de producción se van ensamblando con el marco de la bicicleta hasta lograr el producto terminado.

\section{Construcción del modelo de simulación}

Una vez definidos la estructura del producto y la secuencia del proceso de fabricación, se desarrolló un modelo conceptual, el cual se codificó posteriormente empleando un software de simulación apropiado (Promodel, 2014) para el modelamiento de sistemas de producción, teniendo en cuenta las variables de la situación a representar y sus relaciones en términos de causa- efecto. Igualmente se verificó que el modelo codificado se correspondiera con el modelo conceptual y se validó que el comportamiento del modelo se aproximara al comportamiento del sistema representado. En la Figura 2 se muestra una representación gráfica del modelo de simulación desarrollado. La Figura 3 muestra el flujo del proceso simulado.

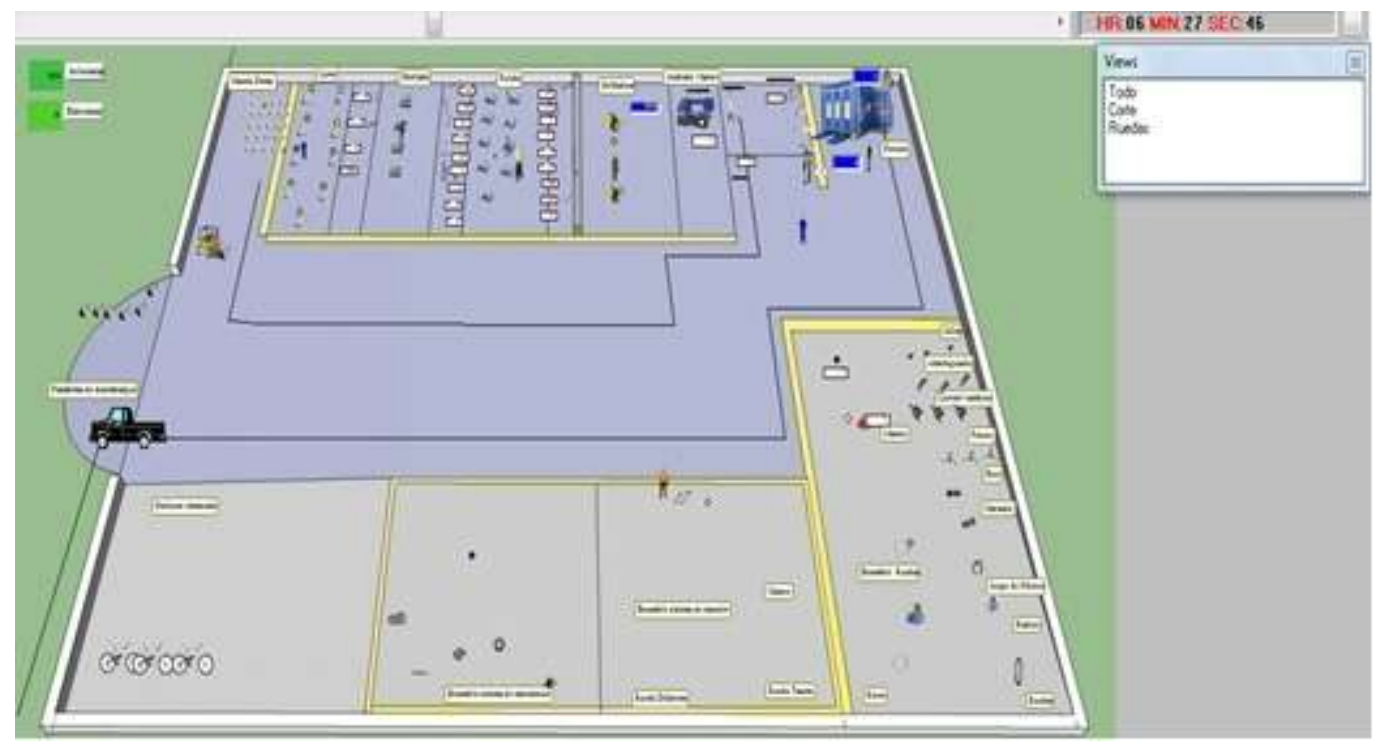

Fig. 2: Representación gráfica del modelo de simulación

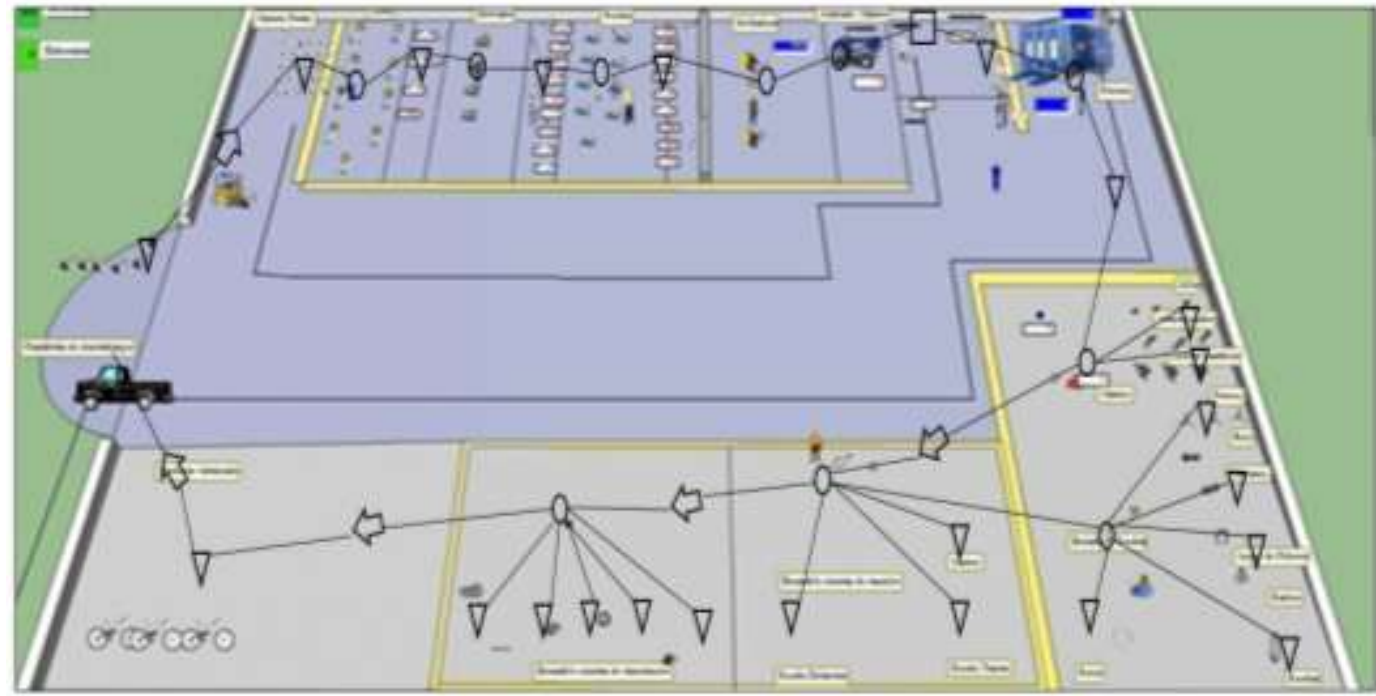

Fig. 3: Representación gráfica del flujo de proceso simulado

\section{Definición del modelo de decisión}

La expresión (1) denota el modelo lógico-matemático simbolizado en la simulación. En este las $m$ variables respuesta (efecto) $y_{i}$ dependen de relaciones lógico/matemáticas de hasta $n_{j}$ variables de decisión $x_{i, j}$ descritas por la función $\mathrm{f}_{\mathrm{j}}\left(\mathrm{X}_{\mathrm{i}, \mathrm{j}}\right)$. La función $f_{j}$ es representada por el modelo de simulación. Los valores de las 
$n_{j}$ variables de decisión $x_{i, j}$ serían los niveles de decisión que estarían directamente relacionados con la medida de desempeño del sistema yi que se quiere estudiar.

$y_{j}=f_{j}\left(x_{i, j}\right)$ donde $1 \leq i \leq n_{j} ; 1 \leq j \leq m$

Las expresiones siguientes (ver Tabla 2) son posibles instancias del modelo de decisión de la expresión (1), con aplicación práctica en la medición de determinado desempeño del sistema de producción de bicicletas descrito. (El termino U denota distribución de probabilidad uniforme con parámetros media y rango medio).

Tabla 2: posibles instancias del modelo de decisión

\begin{tabular}{|c|c|c|c|}
\hline & \multicolumn{2}{|c|}{ Variables de decisión $x_{i, j}$} & Medida de desempeño $y_{j}$ \\
\hline$x_{1,1}$ & Tiempo de corte de tubo superior & $\cup(6,0.6)$ minuto & \multirow{5}{*}{$\begin{array}{c}y_{1} \\
\text { (Tiempo de producción por } \\
\text { unidad con } n_{1}=5 \text { ) }\end{array}$} \\
\hline$x_{2,1}$ & Inventario inicial de marcos & 0 unidades & \\
\hline$x_{3,1}$ & Tiempo de doblado del manubrio & $U(5,0.4)$ minuto & \\
\hline$x_{4,1}$ & Numero de tubos para vainas que llegan & 30 unidades & \\
\hline$x_{5,1}$ & Paros de la cortadora 1 & Cada 5 unidades & \\
\hline$x_{1,2}$ & Tiempo de corte de tubo superior & $\mathrm{U}(6,0.6)$ minuto & \multirow{5}{*}{$\begin{array}{c}y_{2} \\
\text { (Costo Unitario con } n_{2}=5 \text { ) }\end{array}$} \\
\hline$x_{2,2}$ & Inventario inicial de marcos & 0 unidades & \\
\hline$x_{3,2}$ & Tiempo de doblado del manubrio & $U(5,0.4)$ minuto & \\
\hline$x_{4,2}$ & Numero de tubos para vainas que llegan & 30 unidades & \\
\hline$x_{5,2}$ & Paros de la cortadora 1 & Cada 5 unidades & \\
\hline$x_{1,3}$ & Tiempo de corte de tubo superior & $U(6,0.6)$ minuto & \multirow{4}{*}{$\begin{array}{c}y_{3} \\
\text { (Throughput con } n_{3}=4 \text { ) }\end{array}$} \\
\hline$x_{2,3}$ & Tiempo de doblado del manubrio & $U(5,0.4)$ minuto & \\
\hline$x_{3,3}$ & Numero de tubos para vainas que llegan & 30 unidades & \\
\hline$x_{4,3}$ & Paros de la cortadora 2 & Cada 9 unidades & \\
\hline
\end{tabular}

Representación del modelo de decisión en la simulación

La Figura 4 ilustra como a partir de la demanda del mercado, el usuario puede establecer los niveles de operación de las $n_{j}$ variables de decisión $x_{i, j}$ para un determinado escenario y mediante experimentación a través del modelo de simulación mide el desempeño $y_{j}$ con la consiguiente retroalimentación.

\section{Entorno: Demandas del mercado}

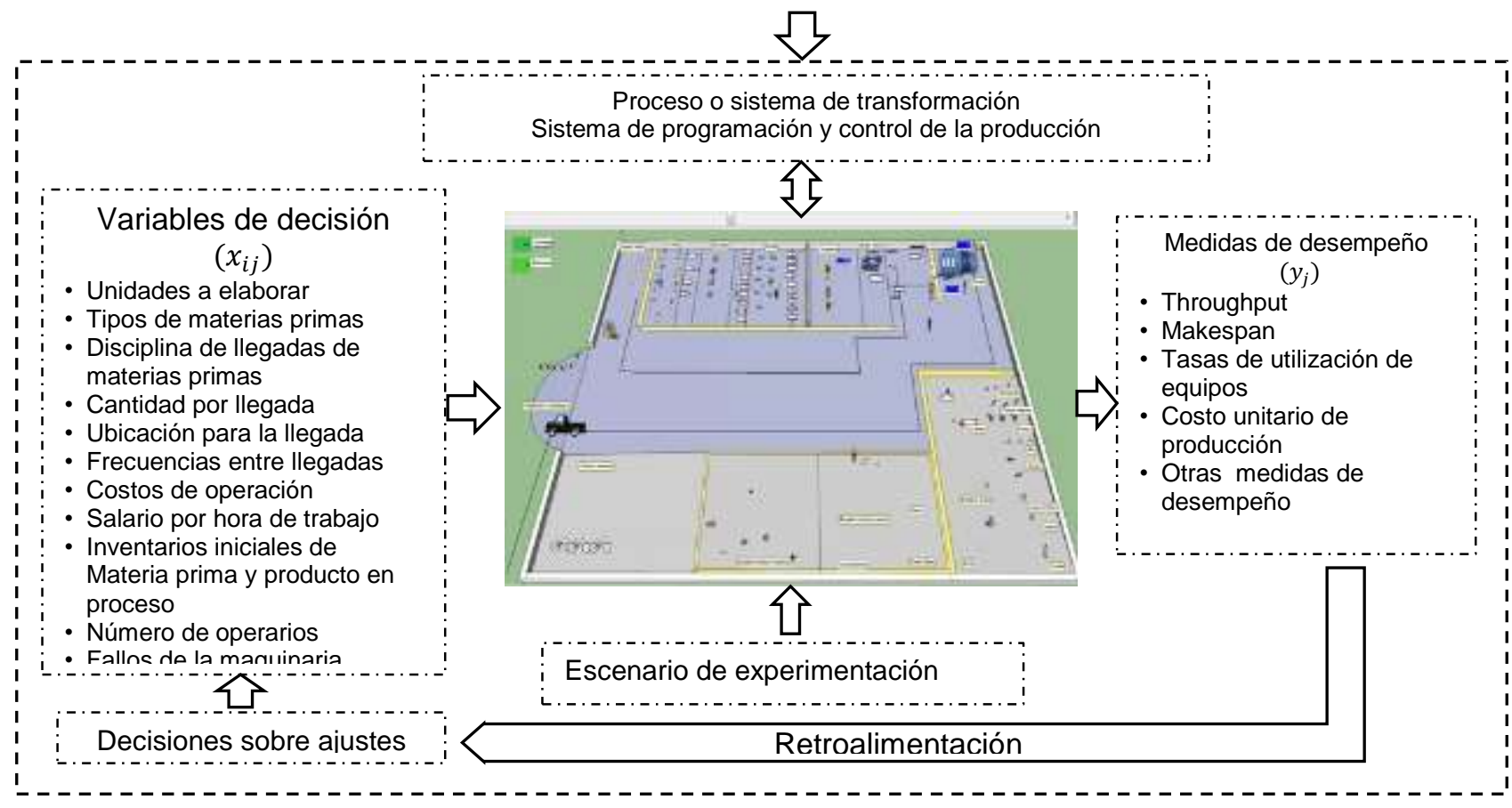

Fig. 4: Representación gráfica de la relación modelo de decisión y modelo de simulación 


\section{Interface usuario-modelo}

En la Figura 5 se muestra la interface desarrollada. Los niveles de las variables de decisión son proporcionados por el usuario a través de hojas Excel. La salida de interés (desempeño del sistema modelado) también es registrada en hojas Excel.
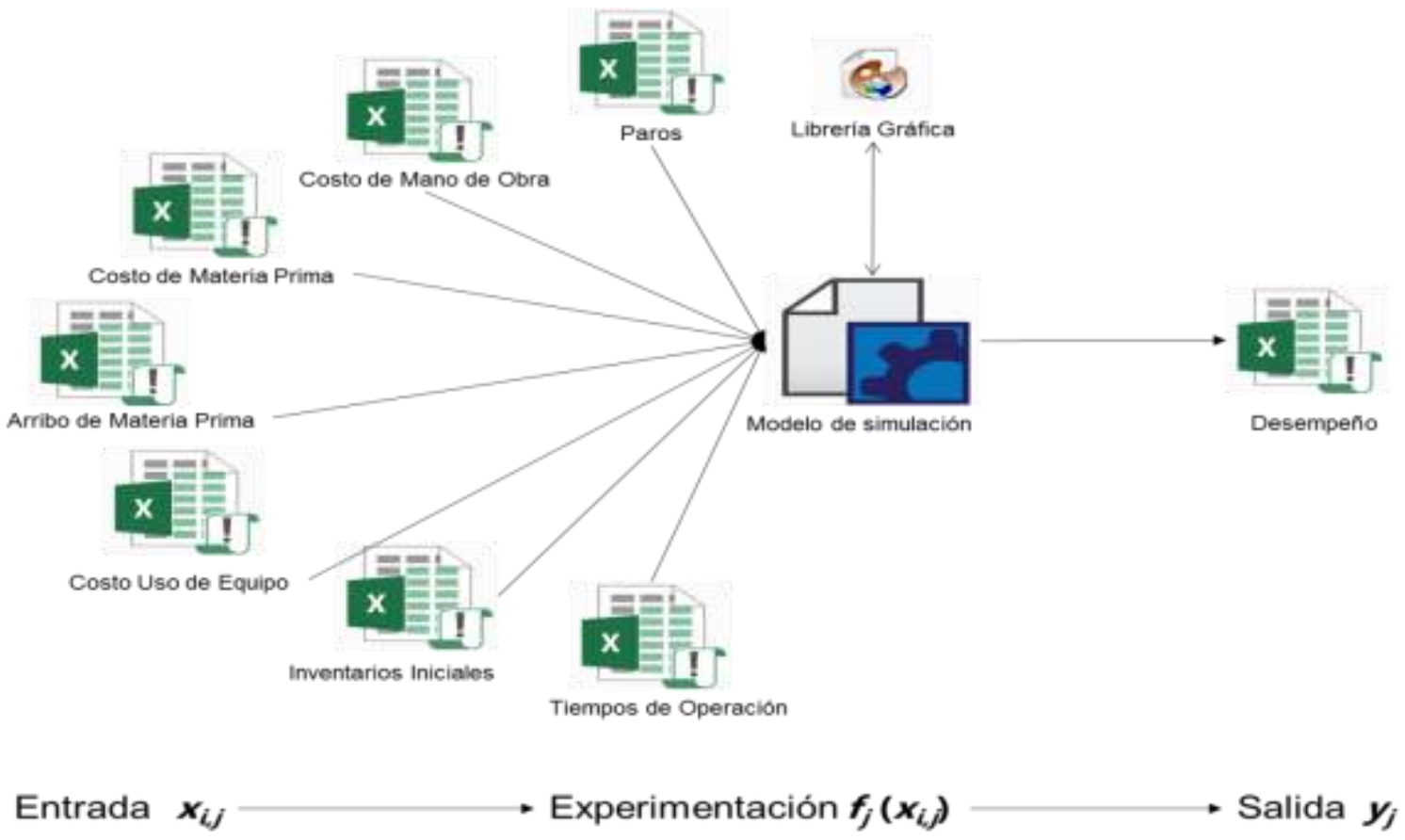

Fig. 5: Esquema general de la interface usuario-modelo

En la Figura 6 se ilustra, por ejemplo, el formato de la hoja Excel que permite fijar las variables de decisión relacionados con el arribo de materias primas (Arribo $\mathrm{mp}$ ).

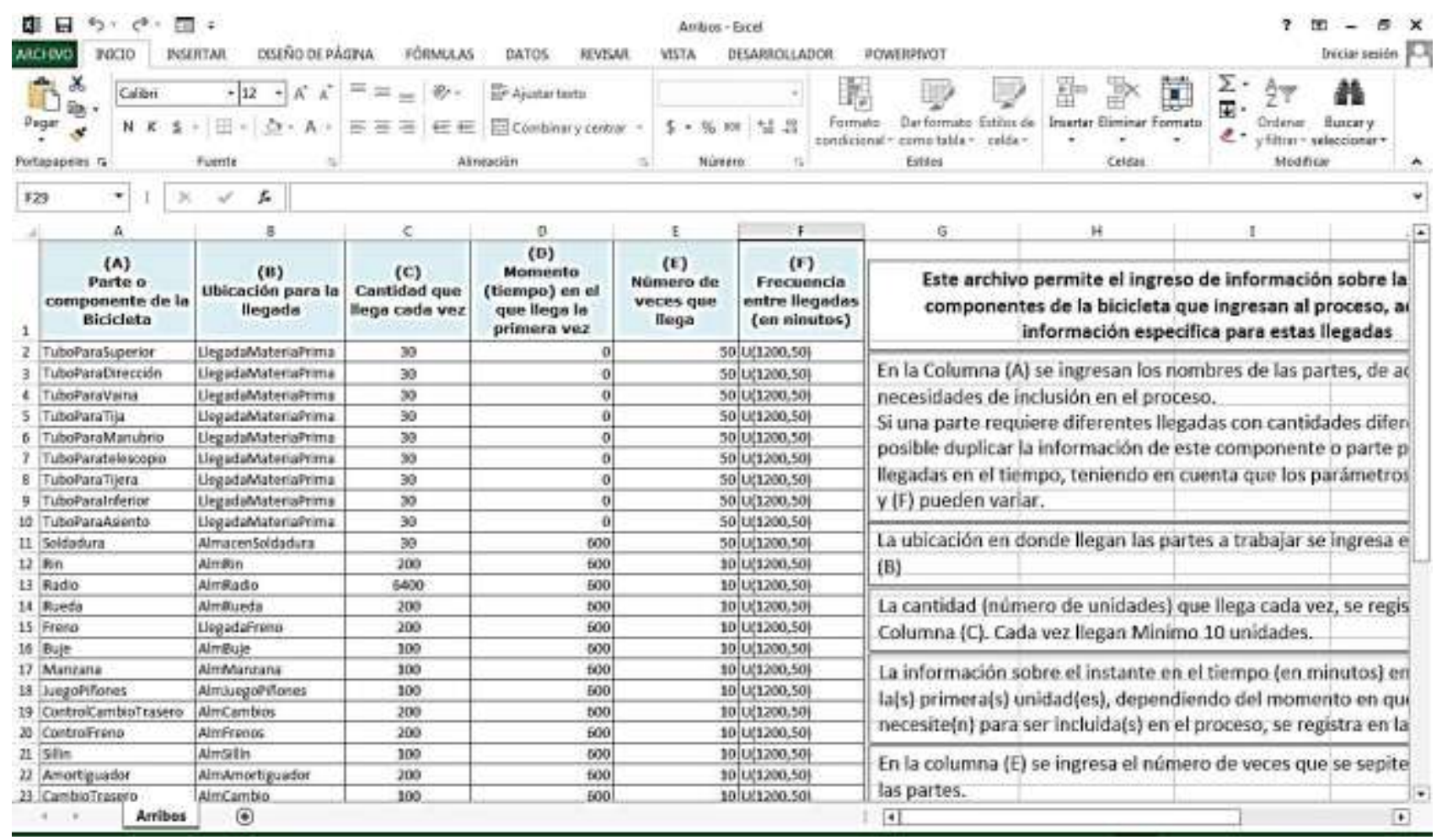

Fig. 6: Interface para fijar niveles de variables de decisión sobre arribo de materias primas 


\section{Experimentación con el modelo}

Son múltiples las instancias del problema que se pueden simular, según se desprende del modelo lógicomatemático representado en la expresión (1). Para efectos de demostración, se considerará una instancia del problema con $m=2$ variables respuesta y $n_{1}=n_{2}=5$ variables de decisión, manteniendo constantes los demás factores. En la Tabla 3 se muestra el significado de cada una de las variables de experimentación a considerar. Al iniciar la simulación del proceso se solicita al usuario el numero $p$ de bicicletas a fabricar, seguidamente se simula su proceso de fabricación a 10 réplicas en cada uno de los diferentes escenarios propuestos.

Tabla 3: Variables respuesta y decisión de una posible instancia del problema

\begin{tabular}{|c|c|l|}
\hline Variable respuesta & & \multicolumn{1}{|c|}{ Descripción de la variable de decisión } \\
\hline \multirow{2}{*}{$\begin{array}{c}y_{1} \\
\text { (Tiempo total para } \\
\text { procesar } p \text { productos: } \\
\text { Makespan) }\end{array}$} & $x_{1,1}$ & Tiempo de corte del tubo para manubrio \\
\cline { 2 - 3 } & $x_{2,1}$ & Tiempo de corte del tubo para asiento \\
\cline { 2 - 3 } & $x_{3,1}$ & Tiempo de corte del tubo parte superior marco \\
\cline { 2 - 3 } & $x_{4,1}$ & Tiempo de corte del tubo parte inferior marco \\
\hline \multirow{4}{*}{$\begin{array}{c}y_{2} \\
\text { (Tasa de utilización } \\
\text { promedio de la } \\
\text { sección de corte })\end{array}$} & $x_{5,1}$ & Tiempo de corte del tubo para dirección \\
\cline { 2 - 3 } & $x_{1,2}$ & Tiempo de corte del tubo para manubrio \\
\cline { 2 - 3 } & $x_{2,2}$ & Tiempo de corte del tubo para asiento \\
\cline { 2 - 3 } & $x_{3,2}$ & Tiempo de corte del tubo parte superior marco \\
\hline
\end{tabular}

\section{RESULTADOS Y DISCUSIÓN}

\section{Instancia del problema a resolver}

Según lo expuesto en la introducción, una instancia del problema a plantearle al discente debe reunir unos requisitos mínimos en el marco del ABP. La Tabla 4, describe la instancia del problema con la cual se experimentará. Dado que por cuestiones de espacio es imposible mostrar todas las variables de decisión y de respuesta que permiten generar diversas instancias del problema, según lo representa la expresión (1), se ha publicado en el sitio www.simulandofabricabicicletas.wordpress.com toda la información sobre el modelo.

Tabla 4. Descripción de la instancia del problema a resolver

Enunciado del problema

Relación con el conocimiento previo de los discentes

Elementos desconocidos del problema que demanden más información

Interés y relevancia para los estudiantes

¿Cuáles han de ser los valores aproximados de los tiempos de corte que permiten obtener simultáneamente un valor apropiado (bajo) de makespan y altas tasas de utilización de los centros de corte al fabricar $p$ bicicletas?

El discente debe haber aprobado un curso básico de gestión de producción y logística. Además estar familiarizado con un ambiente de fabricación tipo flow shop y tener conocimientos básicos sobre control de producción con enfoque push

Debería el estudiante indagar por: tiempos entre fallos de máquinas de corte y los tiempos de reparación, expresándolos en términos de distribuciones de probabilidad. Además preguntarse por la duración de la jornada laboral, tiempos de operación en otras operaciones diferentes al corte, etc.

El interés se da en razón a poder experimentar con un sistema virtual, casi a costo cero. La relevancia reside en trabajar con objetos de estudio propios de la ingeniería industrial (ambiente empresarial)

Objetivos de aprendizaje $\quad$ Aprender conceptos sobre demanda independiente/dependiente; árbol de partes formulados por los estudiantes sobre la(s) materia(s) deseada(s) componentes de un producto; árbol de procesos para fabricar un producto; tiempos de proceso/suministro determinísticos y estocásticos; compras de materia prima; costos de producción; mantenimiento preventivo/correctivo de máquinas de corte

¿El problema refleja la complejidad de los problemas de la vida real?
La complejidad del proceso de fabricación de bicicletas se da en tres dimensiones:

a) Comportamiento aleatorio en tiempos de operación, tiempos entre fallos y tiempos de reparación

b) Alta interdependencia entre las etapas del proceso (ver figura 1)

c) Necesidad de estudiar el comportamiento del proceso durante un periodo de tiempo para resolver el problema (lo que demora hacer $p$ bicicletas bajo ciertos valores de las variables de decisión) 


\section{Simulando escenarios de la instancia del problema}

En la Tabla 5 se muestran dos escenarios posibles de variables de decisión. Los valores de las variables pueden ser determinísticos (escenario 1) o aleatorios (escenario 2). En este último caso, estos se representan mediante distribuciones de probabilidad con sus respectivos parámetros, tales como la uniforme $(\mathrm{U})$, normal $(\mathrm{N})$, etc.

Tabla 5: Escenarios de simulación.

\begin{tabular}{|c|l|c|c|c|}
\hline $\begin{array}{c}\text { Variable } \\
\text { decisión }\end{array}$ & \multicolumn{1}{|c|}{ Descripción de la variable de decisión } & $\begin{array}{c}\text { Unidad de } \\
\text { medida }\end{array}$ & Escenario 1 & Escenario 2 \\
\hline$x_{1, j}$ & Tiempo de corte del tubo para manubrio & Minuto & 5 & $\mathrm{U}(6,0.6)$ \\
\hline$x_{2, j}$ & Tiempo de corte del tubo para asiento & Minuto & 4 & $\mathrm{U}(7.5,0.75)$ \\
\hline$x_{3, j}$ & Tiempo de corte del tubo parte superior marco & Minuto & 5 & $\mathrm{U}(6,0.6)$ \\
\hline$x_{4, j}$ & Tiempo de corte del tubo parte inferior marco & Minuto & 2.5 & $\mathrm{U}(3,0.1)$ \\
\hline$x_{5, j}$ & Tiempo de corte del tubo para dirección & Minuto & 3.1 & $\mathrm{U}(4,0.3)$ \\
\hline
\end{tabular}

\section{Resultados de la experimentación}

En la Tabla 6 se muestran los resultados obtenidos al simular ambos escenarios, en términos de las variables makespan y tasa de utilización promedio de la sección de corte. Se espera que con los resultados de la Tabla 6, incluyendo escenarios adicionales, el estudiante pueda aproximarse a dar solución a la instancia del problema enunciado en la Tabla 4. Igualmente debe considerarse que bajo ABP, no interesa demasiado la solución al problema, pero si al proceso de aprendizaje al buscar esta.

Tabla 6: Resultados variables respuesta en escenarios de simulación 1 y 2

\begin{tabular}{|l|c|c|}
\hline \multicolumn{1}{|c|}{ Variable respuesta } & Escenario 1 & Escenario 2 \\
\hline $\begin{array}{l}y_{1}: \text { (Tiempo total para fabricar 50 } \\
\text { bicicletas: Makespan) }\end{array}$ & 7482 (Minutos) & 6966 (Minutos) \\
\hline $\begin{array}{l}y_{2}: \text { (Tasa de utilización promedio de } \\
\text { la sección de corte ) }\end{array}$ & $86.95 \%$ & $81.22 \%$ \\
\hline
\end{tabular}

\section{Prueba piloto}

Con el fin de evaluar la utilidad didáctica de la herramienta informática desarrollada se realizó una prueba piloto con un grupo de ocho (8) estudiantes del programa de Ingeniería Industrial de la UAM® que hubiesen cursado la asignatura de Ingeniería de la Producción y que tienen pendiente cursar Gestión de la Producción. Para el desarrollo de la prueba se siguieron los siguientes pasos: 1) Se informa que el objetivo de la prueba es evaluar el modelo de simulación como soporte al aprendizaje sobre cómo gestionar un sistema de producción industrial 2) Se dan indicaciones para la instalación de la herramienta. 3) Se instruye sobre el uso del modelo y los archivos de ingreso de datos y salida de información. 4) Se realiza una corrida inicial del modelo, con la información por defecto contenida en los archivos de entrada, a fin que se comprenda el proceso representado. 5) Se realizan algunos ejercicios, con información de entrada propuesta por el docente. 6) Se realizan algunos ejercicios, con información de entrada propuesta por los estudiantes de manera individual con el fin de hacer análisis "¿qué tal si...?". Seguidamente a cada estudiante se le hicieron las preguntas mostradas en la Tabla 7. En la Tabla 8, se muestran las estadísticas sobre las respuestas dadas por los estudiantes a las preguntas de la Tabla 7.

Se connota en general que la herramienta desarrollada ha sido bien evaluada. Puntualmente es de resaltar los siguientes resultados de la prueba piloto: respecto a la pregunta 2 el $87.5 \%$ de los estudiantes muestran que la máxima valoración para la herramienta es 5 , en tanto que el $12.5 \%$ da una calificación de 4 , en conjunto tenemos una calificación global de 4,9 lo que nos indica que los estudiantes están de acuerdo en que el modelo si cumple con las condiciones para representar un sistema de producción. En relación con la pregunta 3, el $75 \%$, califican entre bajo y medio-bajo el grado de dificultad en el ingreso de los datos de entrada, esto se presenta por el grado de familiaridad y facilidad de manejo que ofrece el Excel al ser un paquete utilitario de amplia difusión y dominio por parte de los estudiantes y docentes. Sin embargo se presentan valoraciones alrededor del $25 \%$ que consideran que el ingreso de información de entrada presenta un mayor grado de dificultad. Respecto a la pregunta 4, en su gran mayoría los estudiantes consideran que los resultados para el modelo desarrollado son coherentes con los resultados que se presentarían en un proceso productivo, sin embargo cabe analizar el por qué no lo valoran así la totalidad. 
Tabla 7: Cuestionario aplicado en la prueba piloto

\begin{tabular}{|c|c|}
\hline Enunciado pregunta & Escala de respuesta \\
\hline $\begin{array}{l}\text { Pregunta 1: ¿Cómo evalúa la estructura del modelo, } \\
\text { archivos de entrada de información y archivos de salida? }\end{array}$ & Excelente, Buena, Regular, Mala \\
\hline $\begin{array}{l}\text { Pregunta 2: ¿Cómo califica el modelo elaborado para la } \\
\text { representación de un sistema de gestión de la } \\
\text { producción?, donde } 1 \text { es la calificación más baja y } 5 \text { la } \\
\text { más alta. }\end{array}$ & 1 a 5 \\
\hline $\begin{array}{l}\text { Pregunta 3: Califique el grado de dificultad para el ingreso } \\
\text { de la información de entrada. }\end{array}$ & Bajo; Medio bajo; Medio; Medio Alto; Alto \\
\hline $\begin{array}{l}\text { Pregunta 4: ¿Considera que los datos de salida del } \\
\text { modelo de simulación desarrollado obedecen a los } \\
\text { resultados lógicos de un proceso productivo? }\end{array}$ & Totalmente; Parcialmente; Nada \\
\hline $\begin{array}{l}\text { Pregunta 5: ¿Cómo califica la utilidad de la herramienta } \\
\text { desarrollada para la comprensión de las temáticas de } \\
\text { gestión de la producción? }\end{array}$ & Bajo; Medio bajo; Medio; Medio Alto; Alto \\
\hline $\begin{array}{l}\text { Pregunta 6: ¿Considera que la herramienta es un buen } \\
\text { apoyo para mejorar el aprendizaje de temáticas de } \\
\text { gestión de la producción? }\end{array}$ & Sí; No \\
\hline $\begin{array}{l}\text { Pregunta 7: ¿Considera que la herramienta es una buena } \\
\text { opción para que el docente apoye en ella sus clases y } \\
\text { mejorar los estímulos de aprendizaje hacia los } \\
\text { estudiantes? }\end{array}$ & Sí; No \\
\hline $\begin{array}{l}\text { Pregunta 8: ¿Cómo califica la flexibilidad del modelo de } \\
\text { simulación para la generación de escenarios de } \\
\text { evaluación de producción diferentes? }\end{array}$ & Bajo; Medio bajo; Medio; Medio Alto; Alto \\
\hline Pregunta 9: Sugerencias o comentarios. & Respuesta abierta \\
\hline
\end{tabular}

Tabla 8: Respuestas dadas al cuestionario aplicado en la prueba piloto

\begin{tabular}{|c|c|c|c|c|c|c|c|}
\hline \multicolumn{2}{|c|}{ Pregunta 1} & \multicolumn{2}{|c|}{ Pregunta 2} & \multicolumn{2}{|c|}{ Pregunta 3} & \multicolumn{2}{|c|}{ Pregunta 4} \\
\hline Excelente & $50,0 \%$ & 1 & & Bajo & $37,5 \%$ & Totalmente & $87,5 \%$ \\
\hline Muy Buena & $50,0 \%$ & 2 & & Medio bajo & $37,5 \%$ & Parcialmente & $12,5 \%$ \\
\hline Buena & & 3 & & Medio & $12,5 \%$ & Nada & \\
\hline Regular & & 4 & $12,5 \%$ & Medio Alto & $12,5 \%$ & & \\
\hline Deficiente & & 5 & $87,5 \%$ & Alto & $0 \%$ & & \\
\hline \multicolumn{2}{|c|}{ Pregunta 5} & \multicolumn{2}{|c|}{ Pregunta 6} & \multicolumn{2}{|c|}{ Pregunta 7} & \multicolumn{2}{|c|}{ Pregunta 8} \\
\hline Bajo & & $\mathrm{Si}$ & $100,0 \%$ & Si & $100,0 \%$ & Bajo & \\
\hline Medio bajo & & No & & No & & Medio bajo & \\
\hline Medio & & & & & & Medio & \\
\hline Medio Alto & $12,5 \%$ & & & & & Medio Alto & $25,0 \%$ \\
\hline Alto & $87,5 \%$ & & & & & Alto & $75,0 \%$ \\
\hline
\end{tabular}

Con respecto a la pregunta 5, los resultados muestran que el 100\% de los estudiantes dan una valoración de media alta y alta en la utilidad de la herramienta desarrollada en la comprensión de las temáticas de gestión de la producción, lo que indica que su uso aportaría de manera significativa a la comprensión de las temática propias de esta asignatura y permite presumir que la adopción de ella en la explicación de algunos temas de la asignatura mencionada aportarían al fortalecimiento de conceptos. En relación con las preguntas 6 y 7, el $100 \%$ de los estudiantes valoran positivamente el empleo de la herramienta y su inclusión como soporte en el tratamiento de algunas temáticas de la asignatura; esto concuerda con la presunción hecha desde los postulados teóricos que manifiestan que la evolución de la educación y de la tecnología impactan de manera positiva los procesos educativos, además el $100 \%$ de los estudiantes valoran positivamente el empleo de la herramienta y consideran que la labor docente mediante su inclusión sería más dinámica y motivante para los estudiantes. Finalmente las respuestas a la pregunta 8 , indican que el $75 \%$ de los encuestados consideran que el modelo es altamente flexible en abarcar diversos escenarios de producción industrial. 


\section{CONCLUSIONES}

De los resultados y el análisis expuesto se obtienen la siguientes conclusiones: i) La herramienta desarrollada permitió, de un lado, modelar de un sistema real su variabilidad, interdependencia entre componentes y comportamiento en el tiempo y de otra parte, obtener un modelo de decisión coherente, en términos de variables de decisión que influyen en el desempeño típico de dichos sistemas. ii) En los programas de Ingeniería Industrial, el aprendizaje basado en problemas (ABP), apoyado en herramientas virtuales como la simulación, permite generar tanto por docentes como por discentes, múltiples instancias de un problema que obligan a estos últimos a investigar sobre elementos desconocidos que demanden más información y conocimiento sobre el sistema y/o proceso en el cual se presenta el problema, y iii) se propicia una experimentación con un sistema virtual casi a costo cero y cuya relevancia reside en trabajar con objetos de estudio propios de un ambiente empresarial, involucrando la complejidad inherente a los sistemas de fabricación que los futuros profesionales se encontraran en su desempeño laboral.

\section{REFERENCIAS}

Alejandro, C., Prácticas de Laboratorio de Física General en Internet, REEC: Revista Electrónica de Enseñanza de las Ciencias, ISSN-e: 1579-1513 (en línea;

http://reec.uvigo.es/volumenes/volumen3/REEC_3_2_6.pdf. Acceso: 5 de Febrero de 2014 ), 3 (3), 202-210 (2004)

Álvarez de Zayas, Carlos. Didáctica. La escuela en la vida. Editorial Pueblo y Educación. La Habana. (1999)

AUNIES. La educación superior para elsiglo XXI. Asociación nacional de universidades e instituciones de educación superior. México (2001)

Banks, J.; Carson , J.; Nelson, B. y Nicol, D. Discrete Event System Simulation. 5a Edición. US. Prentice Hall (2009)

Chung. C.A. Simulation Modeling Handbook: A Practical Approach. CRC Press. Boca Raton. (2003)

Ertugrul, N., Towards Virtual Labortories: A survey of LabView-Based Teaching/Learning Tools and Future Trends. International Journal of Engineering Education, 171- 180 (2000)

Feo, R. Orientaciones básicas para el diseño de estrategias didácticas. Tendencias pedagógicas, 220-236. (2010)

Fernández, F., y Duarte, J. El Aprendizaje basado en Problemas como Estrategia para el Desarrollo de Competencias Específicas en Estudiantes de Ingeniería. Formación Universitaria, 6, 29-38. (2013)

Ferreiro, R. Estrategias didácticas del aprendizaje cooperativo. Ed. Trillas, México (2004)

Fredes, C. A.; Hernández, J. P. y Díaz, D. A. Potencial y Problemas de la Simulación en Ambientes Virtuales para el Aprendizaje, Formación Universitaria. 5(1), 45-56 (2012)

Giraldo, J. Simulación de sistemas de producción y de servicios. Manizales: Editorial Blanecolor S.A.S. (2014)

Gómez, V., y Díaz, M. Formación por ciclos en la Educación Superior. Serie Calidad de la Educación Superior No.9. MEN-ICFES (2003)

Gordon, G. System Simulation Prentice Hall. (1978)

Harrell, C., Ghosh. B. Simulation using Promodel, First Edition, United States of America, McGraw Hill. (2004)

Helsel, S., Virtual reality and education, Educational Technology: 32(5), 38-42 (1992)

Holvikiki, J. Learning styles in engineering education: the quest to improve didactic practices. European Journal of Engineering Education, 401-408 (2007)

ITESM, Inst. Tecnol. y de Estudios Superiores de Monterrey. El Aprendizaje Basado en Problemas como Técnica Didáctica. (en línea; 
http://www.rsu.uninter.edu.mx/doc/marco_conceptual/EIMetododeProyectoscomoTecnicaDidactica.pdf. Acceso: 16 de Febrero de 2014). (2000)

Laborí de la Nuéz, B., y Olegordia Aguirre, I. Estrategia educativas para el uso de las nuevas tecnologías de la información y de la educación. Revista Iberoamericana de educación (2011)

Law, A. y Kelton, W. Simulation, modeling and analysis 3a ed. México: Mc GrawHill. (2007)

López Cerezo, J. A., y Valenti, P. Educación tecnologica en el siglo XXI. Revista Polivalencia. (2000)

Molina Alvarez, A. Didáctica de la Ingeniería: Fundamentos teóricos y metodológicos. La habana: Editorial Universitaria Cubana. (2012)

Moran Moguel, C. Estrategias de Incorporación del Aprendizaje Basado en Proyectos en las Instituciones de Educación Superior en Ingeniería len linea en http://www.ai.org.mx/ai/images/sitio/edodelarte/2011/3._estrategia_de_incorporacion_del_aprendizaje_basa do_en_proyectos_en_las_ies_en_ingenieria.pdf. Acceso 12 de mayo de 2014). (2011)

Okutsu, M., DeLaurentis, D., Brophy, S., y Lambert, J. Teaching and Aerospace Engineering Design Course via Virtual Worlds: A Comparative Assessment of Learning Outcomes. Computers and education, 60(1) 288298. (2013)

Ortiz Ocaña, A. Didactica problematizadora y aprendizaje basado en problemas. Bogotá: Ediciones Litoral (2009)

Pozo, J., y Monereo, C. El aprendizaje estratégico. Enseñar a aprender desde el currículo. Madrid: Santillana (1999)

Restrepo, B. Aprendizaje Basado en Problemas (ABP): Una Innovación Didáctica para la Enseñanza Universitaria. Educación y Educadores (2005)

Shannon R. Systems Introduction to the Art and Science of Simulation. Proceedings of the 1998 Winter Simulation Conference (1998)

Torres Salas, M. I. La enseñanza tradicional de las ciencias versus las nuevas tendencias educativas. (en linea; http://revistas.una.ac.cr/index.php/EDUCARE/article/view/1515.Acceso 24 de mayo de 2014), 131 142. (2010)

Troye Nordkvellei, Y. Technology and didactics: historical mediations of a relation. Journal of curriculum studies, (en línea; http://web.a.ebscohost.com.ezproxy.unal.edu.co/ehost/pdfviewer/pdfviewer?sid=bdbfd4c1-ac1a-409f-869e039e677eaae8\%40sessionmgr4001\&vid=2\&hid=4106. Acceso: 22 de mayo de 2014). (2004)

UNESCO. Medición de las tecnologías de la información y la comunicación (TIC) en educación. Montreal: UNESCO Institute for Statistics (2009)

Vacca, V., Caicedo, E., y Ramirez, j. Herramienta Remota de Cálculo y Multiusuario para el Aprendizaje Basado en Problemas Usando Matlab. Revista Facultad de Ingeniería, 158 -169. (2011)

Vizcarro, C., Juárez E., La metodología del aprendizaje basado en problemas. (en línea: www.ub.edu/dikasteia/LIBRO_MURCIA.pdf. Acceso 22 de mayo de 2014) (2007) 